\title{
LA/S AGENDA/S DE GÉNERO SINDICAL EN MENDOZA, ARGENTINA: 2016-2020
}

\author{
Patricia Gorri ${ }^{1}$
}

Patricia Lecaro ${ }^{2}$

\begin{abstract}
RESUMEN: El movimiento sindical vuelve a ser objeto de estudio para las Ciencias Sociales acentuando diferentes dimensiones de estudio respecto de los años 90, emergiendo en los últimos cinco años el tema de la marea feminista en el ámbito sindical. Presentamos este trabajo sobre la/s agenda/s de género en los sindicatos estatales de Mendoza - Argentina - las cuales visibilizan la reconfiguración política y económica que durante el periodo 2016-2020 en el que gobierna la alianza Cambiemos, de claro corte conservador en sus políticas públicas lo que conllevó un ajuste en términos neoliberales que afectó en su mayoría a los/las trabajadores, influyendo en su organización, representación, movilización y agendas. Este trabajo es un primer acercamiento en nuestro proceso de investigación a la agenda de género sindical y a su vez nos permite reflexionar sobre los dilemas que enfrenta el compromiso académico y la investigación crítica al abordar nuestro objeto de estudio desde lo conceptual y en su práctica política sindical. El trabajo está estructurado de la siguiente manera: en primer lugar, hacemos un recorrido conceptual que da cuenta de aquellas categorías que consideramos más fructíferas para el análisis e interpretación de nuestro objeto de estudio. En este sentido retomamos el camino teórico recorrido por referentes en el tema de feminismo y sindicalismo (GAMBA, 2008; ASPIAZU, 2015; GOLDMAN, 2018; LENGUITA, 2017, 2019, 2020; NATALUCCI y REY, 2018; DIAZ y SOCOLOVSKY, 2020; GOREN y PRIETO, 2020). Con estas herramientas teóricas visitamos conceptos y describimos la militancia feminista en sindicatos estatales de Mendoza intentando dar cuenta de sus prácticas, discursos y agendas. En un segundo momento, hacemos un abordaje metodológico mediante la aproximación a los datos secundarios sobre trabajo y género en Mendoza que nos permiten dimensionar cuantitativamente el fenómeno bajo estudio. En tercera instancia, llevamos cabo la producción de un mapeo de los sindicatos provinciales respecto a las estructuras institucionales para observar si es visible en su organización interna la perspectiva de género. Por último, reflexionamos sobre los límites y desafíos del proceso de investigación en tiempos de pandemia.
\end{abstract}

PALABRAS CLAVES: Sindicatos estatales. Género. Agenda/as de género. Mendoza.

1 Graduada en Ciencia Política y Administración Pública por la Universidad Nacional de Cuyo, Argentina. Magister en Ciencia Política en la Universidad Internacional de La Rábida. Docente de Teoría Política y de Estado, Gobierno y Sociedad. Investigadora en la Fac. Ciencias Políticas y Sociales de la UNCuyo, Argentina. E-mail de contato: pgorri@gmail.com.

2 Graduada en Sociología por la Universidad Nacional de Cuyo, Argentina. Magister en Estudios Latinoamericanos por la Universidad Nacional de Cuyo. Docente de Estructura Social Argentina y Procesos Sociales Contemporáneos. Investigadora en la Fac. Ciencias Políticas y Sociales de la UNCuyo, Argentina. E-mail de contacto: patolecaro@yahoo.com. 


\section{INTRODUÇÃO}

El trabajo se inscribe en una línea de investigación centrada en el estudio del sindicalismo estatal en la Provincia de $\mathrm{Mendoza}^{3}$ en particular conocer la/s agenda/s de género de los sindicatos estatales desde 2016. El trabajo que presentamos es el primer avance de investigación de un nuevo proyecto, focalizando nuestra descripción y análisis en dos gremios estatales - los que reúnen más trabajadoras/es estatales - Asociación Trabajadores del Estado (ATE) y Sindicato de Trabajadores de la Educación (SUTE).

La relación Estado provincial/ sindicatos estatales se ha desarrollado en general de manera conflictiva y sinuosa sobre todo en el periodo 2015-2019. Sin embargo, la gestión del ex Gobernador de Mendoza Alfredo Cornejo (20152019) logró disciplinar al otrora fuerte poder sindical por sus fortalezas políticas apoyada en primer lugar en el consenso que logró en la población; y en segundo lugar, por el debilitamiento y fragmentación de las cúpulas sindicales, explicada en parte por las rupturas acontecidas por la adhesión a alguna de las dos Central de Trabajadores del Estado (CTAs) y las cercanías o disputas con el peronismo y con el Kirchnerismo.

En este contexto, se comenzó a visibilizar la "marea sindical" (GOLDMAN, 2018), el movimiento de mujeres reapareció y se presentó como un emergente transversal, que si bien no es novedoso en sus demandas, sí lo es en la fortaleza y dinamismo con el cual irrumpe en el escenario social y político mendocino.

El trabajo está estructurado de la siguiente manera: en primer lugar, hacemos un recorrido conceptual que da cuenta de aquellas categorías que consideramos más fructíferas para el análisis e interpretación de nuestro objeto de estudio. En este sentido retomamos el camino teórico recorrido por referentes en el tema de feminismo y sindicalismo (SCOTT, 1996; ALBERDI, 1999; GAMBA, 2008; ASPIAZU, 2015; GOLDMAN, 2018; LENGUITA, 2017, 2019, 2020; NATALUCCI y REY, 2018; DÍAZ y SOCOLOVSKY, 2020; GOREN y PRIETO, 2020). Con estas herramientas teóricas visitamos conceptos y describimos la militancia feminista en sindicatos estatales de Mendoza intentando dar cuenta de sus prácticas, discursos y agendas. En un segundo momento, hacemos un abordaje metodológico mediante la aproximación a los datos secundarios sobre trabajo y género en Mendoza que nos permiten dimensionar cuantitativamente el fenómeno bajo estudio. En tercera instancia, llevamos cabo la producción de un mapeo de los sindicatos provinciales respecto a las estructuras institucionales para observar si es visible en su organización interna la perspectiva de género. Por último, reflexionamos sobre los límites y desafíos del proceso de investigación en tiempos de pandemia.

3 La agenda sindical en un contexto de ajuste: estrategias de acción. La marea verde en los sindicatos. Proyecto de Investigación SIIP-UNCUYO 2019-2021. Mendoza es un gobierno subnacional ubicado al oeste de la República Argentina, es la $5^{\circ}$ provincia según el nivel de población (1.738 habitantes, Censo 2010). Cuenta con 99 mil empleados públicos (datos a setiembre 2019). 
El trabajo es parte de una línea de investigación que venimos desarrollando hace seis años sobre los sindicatos en Argentina y Mendoza. Es resultado de la profundización de interrogantes y temas emergentes que quedaron abiertos en el proceso de investigación anterior. En nuestro recorrido vimos aparecer la marea feministas en los sindicatos y en las calles lo cual nos impulsó a redefinir nuestro interés en la convergencia de sindicalismo y feminismo.

$\mathrm{Al}$ artículo lo elaboramos recuperando líneas de investigación respecto a género y sindicalismo en Argentina (ABAL MEDINA, 2012; ASPIAZU, 2015; GOLDMAN, 2018; LENGUITA, 2017, 2019, 2020; NATALUCCI y REY, 2018; DÍAZ y SOCOLOVSKY, 2020; GOREN y PRIETO, 2020) y producciones propias (GORRI y LECARO, 2017, 2018, 2019). Desde el ámbito de los relevamientos estadísticos en Argentina aparecen Informes, estudios sobre género y trabajo, trabajo productivo y reproductivo, concretamente la Encuesta Permanente de Hogares (INDEC) ${ }^{4}$ que incorpora un apartado sobre Uso del tiempo. A ello se suman, algunos intentos de recolección y sistematización de los propios sindicatos respecto del tema.

Asi, nuestro interés y los datos disponibles nos permiten elaborar anticipaciones de sentido donde la irrupción de la marea feminista atraviesa a todos los sindicatos, interpelándolos en sus prácticas y los sacude en su organización. Evidencian que el espacio sindical es un campo de lucha no solo externo contra las patronales, sino también un campo de lucha interno por el lugar de la mujer en espacios de decisión y de instalación de agenda de género en las luchas y conquistas.

Es necesario y urgente comprender los rasgos del nuevo tiempo y la/s agenda/s de género que visibilizan desigualdades históricas en las tareas de cuidado, déficits de condiciones laborales con perspectiva de género, que implosionan en un contexto incierto de crisis económica que data ya de mediano plazo y una crisis sanitaria desatada por la epidemia mundial de Covid19 en 2020 que atraviesa, desdibuja y en muchos casos arrasa la vida laboral, personal y familiar.

\section{GÉNERO, AGENDAS Y POLITICIDAD CON ENFOQUE DE GÉNERO}

\subsection{LO CONCEPTUAL EN CONTEXTO}

En este apartado nos interesamos por las categorías conceptuales que nos aportan elementos y dimensiones para reflexionar respecto a la inclusión de

4 El INDEC es el Instituto Nacional de Estadísticas y Censos de la República Argentina e implementa desde los 90' la Encuesta Permanente de Hogares a través de la cual se relevan diferentes módulos referidos a condiciones de vida, laborales, sociales, económicas de la población argentina y en 2020 comienza a relevar las tareas de cuidado. 
la dimensión de género en el campo de los estudios del mundo del trabajo ${ }^{5} \mathrm{y}$ en el ámbito sindical especialmente. Consideramos que la teoría nos permite una decodificación y reinterpretación de las demandas y conformación de la/s agenda/s de genero sindicales.

La perspectiva de género opta por una concepción epistemológica que se aproxima a la realidad desde las miradas de los géneros y sus relaciones de poder. Sostiene que la cuestión de los géneros no es un tema a agregar como si se tratara de un capítulo más en la historia de la cultura, sino que las relaciones de desigualdad entre los géneros tienen sus efectos de producción y reproducción de la discriminación, adquiriendo expresiones concretas en todos los ámbitos de la cultura: el trabajo, la familia, la política, las organizaciones, el arte, las empresas, la salud, la ciencia, la sexualidad, la historia. (ALBERDI, 1999, p. 11).

Según la clásica definición de género de Joan Scott (1996, p. 289) "el género es un elemento constitutivo de las relaciones sociales basadas en las diferencias que distinguen los sexos, y (...) es una forma primaria de relaciones significantes de poder".

Si bien el concepto de patriarcado nos brinda un marco para la comprensión y explicación de la situación de las mujeres, resulta insuficiente para problematizar y comprender posiciones y relaciones políticas, sociales, culturales, económicas de las mujeres en tramas históricas concretas.

En los últimos años se produjo un importante avance en las ciencias sociales, al incorporarse los denominados estudios de la mujer como un nuevo paradigma. El género, como categoría social, es una de las contribuciones teóricas más significativas del feminismo contemporáneo. Esta categoría analítica surgió para explicar las desigualdades entre hombres y mujeres, poniendo el énfasis en la noción de multiplicidad de identidades. Lo femenino y lo masculino se conforman a partir de una relación mutua, cultural e histórica, que no remite al sexo de los individuos sino los conductas consideradas femeninas o masculinas (GAMBA, 2008).

La perspectiva de género implica reconocer: las relaciones de poder que se dan entre los géneros que en general son favorables a los varones como grupo social y discriminatorias para las mujeres; que dichas relaciones han sido constituidas social e históricamente y son constitutivas de las personas; que las mismas

5 La visión neoliberal considera al trabajo como un bien que se intercambia en el mercado. Este es un espacio que organiza la producción y la distribución a través de la relación de intercambio: salario prestación. como en otros mercados existe una relación de competencia, los oferentes y demandantes tienen una determinada posición desde la cual pueden tejer diversas estrategias. Entendemos por mundo del trabajo, siguiendo a economía Feminista, un espacio universal donde nos realizamos los seres humanos. 
atraviesan todo el entramado social y se articulan con otras relaciones sociales, como las de clase, etnia, edad, preferencia sexual y religión (ALBERDI, 1999, p. 10).

Siguiendo a Susana Gamba (2008) la categoría de género es una definición de carácter histórico y social acerca de los roles, identidades y valores que son atribuidos a varones y mujeres e internalizados mediante los procesos de socialización.

La comprensión de las agendas sindicales de género implican una reflexión crítica situada histórica y socialmente, develando las relaciones asimétricas sociales y de poder - pero no solo de género sino articulándolas con edad, estado civil, clase social y rama de actividad laboral.

En los últimos veinte años en nuestro país se producen cambios significativos en el mercado laboral: las tasas de desempleo de fines de los $90^{\prime}$ provocan una crisis político, social y económica sin precedentes; desde 2003 hasta 2015 se vive un período marcado por un escenario de gobiernos progresistas en América Latina y en nuestro país se toman decisiones de recuperación económica con un Estado presente que intentan recomponer el crecimiento económico.

En este contexto, como sostiene Paula Abal Medina (2012), uno de los pilares de la política sindical del gobierno kirchnerista ha sido la relegitimación de las organizaciones sindicales como parte necesaria del régimen político postconvertibilidad. Esta relegitimación institucional se llevó adelante a través de lo que podríamos resumir bajo la fórmula "paritarias + topes salariales", es decir, la combinación entre la restitución del ámbito de negociación colectiva (debilitado al extremo durante los años $90^{\prime}$ ) y un fuerte arbitraje gubernamental directo a través de instancias (más o menos formalizadas) de "pacto social", como el Consejo del Salario o los topes de aumento salarial acordados directamente con las direcciones sindicales oficialistas.

En 2015, asumió la presidencia Mauricio Macri y desarrolló un proyecto económico político que "ni siquiera representa los intereses del capital más concentrado sino del capital especulativo, es decir, es el proyecto de los grupos que acumulan sobre la base de la renta: la renta agropecuaria, la renta minera, la renta financiera" (VILAS, 2017). Las medidas del gobierno de Cambiemos comenzaron con: la reducción de aranceles sobre los derechos de exportación de soja (del 35\% al 30\%) y sus derivados (del 32\% al 27\%); eliminación de retenciones a cereales y productos agroindustriales; devaluación del $40 / 50 \%$; modificación de los controles cambiarios (puede comprarse libremente un monto que no supere los dos millones de pesos argentinos mensuales).

El mega ajuste en el gasto público se expresó en medidas económicas, políticas y sociales que fomentaron la especulación vía plazos fijos; la Reforma del Estado; el ajuste social renombrado con el eufemismo del sinceramiento de la economía ${ }^{6}$ la estigmatización del Estado y los/as trabajadores públicos como origen y fuente de todos los males; la vuelta al endeudamiento y el retorno al

6 En 2016, Mauricio Macri presenta una serie de medidas que las enmarca en un "sinceramiento de la economía” como un camino hacia el progreso. Para ampliar el tema ver Informe CEPA, 2018. 
financiamiento vía Fondo Monetario Internacional (FMI), entregando los resortes de la economía argentina a sus recetas e imposiciones. Con el FMI se definieron topes de inflación; recorte de transferencias a las provincias y a la masa salarial de los/as trabajadores estatales; aumento de tarifas; la devaluación de la moneda de cambio "flotante" -; incremento de la tasa de interés y desregulación del mercado financiero; eliminación de los controles de importación y reemplazo por licencias automáticas y no automáticas; liberalización del precio de los combustibles; incremento de tarifas en servicios como luz, agua, gas, aumento del combustible. La recesión y una inflación cercana al $45 \%$ para noviembre de 2018 fueron los resultados más palpables para las/os y las trabajadoras/es argentinos del sinceramiento.

La acción sindical no quedó ajena a las conflictividades que generó esta situación económica. Como señala Paula Lenguita (2017, p. 28) el cambio de rumbo económico adoptado por la coalición conservadora que gobernaba el país provocaba cada vez más descontento popular imponiendo el interrogante sobre el rol sindical en la orientación de ese descontento popular. También, la conflictividad socio laboral se vio atravesada por reclamos de género, por la irrupción de mujeres en las calles reclamando por la legalización del aborto, violencia de género, laboral, femicidios y travesticidios.

Las calles y espacios laborales fueron escenario de movilización: desde el Ni Una Menos (primer convocatoria realizada a través de redes sociales el 3 de junio de 2015), la movilización de cada 8 de marzo con el paro internacional de mujeres (desde 2016), la organización y movilización por el aborto legal, hasta las movilizaciones de trabajadoras en sus sindicatos ante reclamos salariales, conforman una oportunidad para aquellas organizaciones que tienen mayores dificultades de organización y visibilización para influir en los poderes públicos, en el mundo del trabajo o en los mismos espacios de militancia como organizaciones sociales, partidos políticos, sindicatos.

\subsection{LA MILITANCIA FEMINISTA EN SINDICATOS}

Como señala Tali Goldman (2018) hay tres procesos que son ineludibles para pensar la militancia feminista en los sindicatos en Argentina:

Uno fue la reactivación del mundo del trabajo entre 2003 y 2015, que creó empleos y amplió la base de las mujeres trabajadoras, aunque esos puestos no hayan sido necesariamente ocupados de modo equitativo por varones y mujeres. El segundo fue la creación de un imaginario en torno

7 El gobierno de Cambiemos comenzó en el año 2016 haciendo un culto del tipo de cambio flotante y bajo, lo cual significa que el valor de la moneda es fijado por el mercado sin intervención de las autoridades monetarias, como es el Banco Central de la República Argentina. 
a la democratización construido durante el kirchnerismo, que implicó, entre otras cuestiones y leyes, el matrimonio igualitario, la identidad de género, la jubilación para las amas de casa y la lucha contra la violencia de género que se instaló definitivamente en el espacio público a partir de la experiencia de Ni Una Menos en junio de 2015. Y el tercero es el proceso de revitalización sindical que se ha pensado desde las cúpulas sindicales, debido al mejoramiento de las negociaciones paritarias y de convenios colectivos, del incremento de la afiliación, del surgimiento de movimientos de las bases (GOLDMAN, 2018).

La revitalización de género en los sindicatos abrió la ventana de oportunidad para mujeres y colectivos disidentes de visibilizar y organizar la acción colectiva con perspectiva de género. Para nosotros es (re)pensar una politicidad femenina con especificidades de construcción y acción política en los sindicatos y que implica una reflexión teórica y práctica. Siguiendo a Nair Calvo (2002) definimos politicidad como:

Las dimensiones referidas a las sensibilidades políticas de las/os actoras/ es, a sus creencias, a sus actitudes y a sus formas de relacionarse con los debates y las decisiones de la esfera pública. El término politicidad se halla en estrecha relación con el concepto de cultura política. Consideramos que la noción de politicidad permite una aproximación más conveniente a las cuestiones referidas a la cultura política. Si ponemos en relación ambos conceptos, la politicidad da cuenta de la forma en que la cultura política es incorporada en (y por) las/os actores. De forma tal que la politicidad encuentra una referencia más inmediata en las prácticas que genera, en el cómo se constituye la cultura política (AQUÍN, 2018, p. 10).

Desde la observación de las prácticas buscamos analizar los significados y relaciones en torno a la política de las trabajadoras dirigentes en sindicatos cuando ponen en juego sus estrategias de presentar agenda de género en el marco de sus organizaciones de pertenencia. Estrategias entendidas como líneas de acción (BOURDIEU, 1995, p. 89) objetivamente orientadas que los agentes sociales construyen sin cesar en la práctica y que se definen en el encuentro entre el habitus y una coyuntura particular del campo. Tales estrategias deben ser comprendidas tanto desde las estructuras de oportunidad como desde los contenidos de significación que atribuyen las mujeres a las relaciones que establecen con sus referentes sindicales y otros actores como son otros sindicatos u organizaciones sociales (NUM), líderes nacionales o la intersindical de mujeres y con la patronal. Consideramos que las trabajadoras en lo sindicatos construyen un sentido de politicidad en y a través de las relaciones mencionadas. Porque esto influye en cómo se construye la cultura política con enfoque de 
género en los sindicatos, cuáles son los rasgos de esta politicidad y cómo (re) significan lo que es "hacer política".

Metodológicamente, para realizar una primera lectura comprensiva de la politicidad con enfoque de género en los sindicatos, describimos la situación actual de los sindicatos bajo estudio - ATE y SUTE- ${ }^{8}$ y sus dirigentes en relación al tema bajo estudio (agenda de género); identificamos las mujeres que cumplen un rol en torno a este tema y caracterizamos la participación en acciones de construcción de agenda de género.

El Sindicato Único de Trabajadores de la Educación (SUTE), es el sindicato con mayor cantidad de afiliados/as, en las elecciones del 15 de junio del 2017 el padrón daba cuenta de 25.877 afiliados/as que estaban en condiciones de sufragar. El Sindicato es conducido por Sebastián Henríquez (Frente Unido de Resistencia Sindical - FURS-) que proviene de un sector más ligado a la izquierda mendocina y quienes logran desbancar de la conducción a la histórica "agrupación celeste", de orientación peronista y desde la llegada al poder del kirchnerismo fuertemente identificado con el Gobierno Nacional. Conducido institucionalmente entre 1998-2008 por Gustavo Maure, como secretario general celeste, extendió su conducción política hasta el 2017. Desde la llegada de Cambiemos al gobierno de la Provincia, el sindicato docente es uno de las más afectado, desacreditado por el discurso oficial y por la aplicación del polémico ítem aula ${ }^{9}$. Los cambios en la conducción del sindicato más grande de Mendoza pueden ser claramente interpretados como una actitud defensiva por parte de los/as trabajadores de la educación. Aunque cabe señalar que los sondeos de opinión política reconocen en el perfil de clase media de la docencia mendocina parte importante del electorado de Cambia Mendoza ${ }^{10}$.

La Asociación Trabajadores del Estado (ATE) cuenta con aproximadamente 18.000 afiliados/as, distribuidos en diversos ámbitos del empleo público: administración central, salud, parques y bosques y también de los/as trabajadores municipales. Más del $60 \%$ de ATE está formado por mujeres. Actualmente lo conduce Roberto Macho, alineado con el sector verde de ATE nacional (dirigida por Pablo Michelli). Comienza su participación sindical como delegado del Hospital Lencinas de Mendoza, para convertirse en el 2003 en secretario gremial de Vicente Frezza y adjunto de Raquel Blas. Roberto Macho asume el 6 de noviembre 2015, incorporando a nuevos militantes con origen en las nuevas

8 Para ampliar este tema ver Gorri, P y Lecaro, P (2020) Sindicatos y gobierno: relaciones, dilemas, tensiones con el nuevo gobierno. La intersindical en Mendoza 2015-2018. Informe final de investigación (SECyT UNCuyo 06/F024). Mendoza, Universidad Nacional de Cuyo. Facultad de Ciencias Políticas y Sociales. https://bdigital.uncu.edu.ar/objetos_digitales/15056/informefinalgorrisindicatosygobierno.pdf.

9 Îtem aula es un ítem salarial de productividad implementado por la Dirección General de Escuelas para mejorar la calidad educativa (Decreto provincial 228/16), lo cual fue vivido como un castigo por los/as trabajadores de la educación ya que se descontaba una parte del sueldo aun ante licencias por enfermedad.

10 Cambia Mendoza es un frente político electoral conservador conformado por la Unión Cívica Radical y el PRO. Siguen la línea de Mauricio Macri. 
áreas de expansión de ATE que son organismos nacionales y municipales. Macho se autodefine como de orientación Justicialista. En las elecciones de 2019, asume como secretaria adjunta Adriana Iranzo.

Tanto SUTE como ATE han desarrollado en los últimos tres años una agenda de género específica, no relacionada con las áreas de turismo o familia, asistencia social como se hacía en otros momentos. Sin embargo, en el SUTE donde la mayoría de las trabajadoras afiliadas son mujeres históricamente ha sido conducido por un varón; actualmente no hay una mujer como secretaria adjunta y cuenta desde hace un año con la Prosecretaría de DDHH y Género.

\subsubsection{AGENDA SINDICAL EN SUTE (2016-2020)}

En la provincia de Mendoza son más de 75.000 las y los trabajadoras/es de la educación de los cuales un $80 \%$ son mujeres. En el Sindicato Único Trabajadores de la Educación (SUTE) la agenda en 2016-2018 gira en torno al reclamo por el ítem aula, las tareas de cuidado que no contempla este ítem y un paso fundamental que se pide en las paritarias es la reglamentación de la ley 8.806/15 de Licencia por violencia de género.

En 2019, desde la Prosecretaría de DDHH y Género reclaman aplicación efectiva de Educación Sexual Integral en las escuelas; la reglamentación de la ley de violencia de género; trabajo, salario digno, una vida libre de violencia para todas las trabajadoras. Desde las consejerías departamentales y la misma Prosecretaría acompañaron ese año a trescientas mujeres en situación de violencia. Asimismo, reconocen que las mujeres cargan no sólo, con el trabajo en la escuela sino con la mayor parte del trabajo en el hogar, ya sea con el cuidado de los/as hijos/as, o bien, cuidado de familiares enfermos/as. Esto visibiliza la desigualdad y de la misma manera el Ítem Aula constituye una forma de violencia que afecta a las mujeres particularmente.

El trabajo sobre la reglamentación de la ley de Licencia por Violencia de Género (sancionada en 2015) para las trabajadoras de la educación se lleva a cabo en una Comisión Paritaria Mixta entre el SUTE y el Gobierno. Virginia Pescarmona y Natalia Naciff (2020) relatan una cronología de la mesa paritaria para la reglamentación de la ley en la cual se señalan algunos aspectos que son importantes en el mundo del trabajo. Por ejemplo, luego de cuatro instancias sin definiciones concretas e incluso la suspensión de una reunión porque el gobierno se olvidó de asistir no se ha avanzado. Si bien algunas cuestiones quedan reducidas a trámites burocráticos, la discusión es más profunda:

(...) desde el gobierno se solicita una denuncia penal o la orden de restricción y una certificación médica o psicológica para aceptar la licencia, nuestra propuesta es que las compañeras puedan presentar 
una declaración jurada y que sea suficiente para acceder a este derecho, es un "Yo sí te creo" hacia la administración pública (...). Otro de los criterios fundamentales es la no re victimización, la autonomía y el consentimiento de la compañera en todos los pasos del proceso; lo cual lleva a otra discusión profunda en relación al ámbito en el cual se otorgan las licencias: la violencia de genero se considera una enfermedad o una licencia especial (PESCARMONA y NACIFF, 2020). ${ }^{11}$

En setiembre entra en agenda otro tema fundamental que afectan derechos de toda la comunidad educativa como es el envío del proyecto de reforma de la ley de educación por parte del ejecutivo provincial y el llamado a un congreso pedagógico. Si bien está en plena discusión, en lo que respecta a nuestro tema la carencia de una mirada de género, de reconocimiento de las tareas de cuidado son la marca de este proyecto de ley.

\subsubsection{AGENDA SINDICAL EN ATE (2018-2020)}

Además de las luchas por el salario, apertura paritarias, paros frente a políticas de ajuste y tarifazos, movilizaciones ante las elecciones otro tema emerge con fuerza: la/s agenda/s feminista. La visibilización de temas de género en acciones colectivas masivas a nivel nacional e internacional, como \#NiUnaMenos, el 8M paro internacional de mujeres y la disputa por posiciones en la conducción de los sindicatos.

En 2019, organizado por Asociación Trabajadores del Estado (ATE) uno de los sindicatos de los/as trabajadores públicos más grandes junto al de educadores en Mendoza-, realizó el Primer Encuentro Nacional de Mujeres Líderes Sindicalistas y de las Organizaciones Sociales para compartir problemas, conflictos, reclamos, posturas, proyectos, políticas y soluciones, dentro de perspectivas de género e inclusión que ayudarán a conformar un documento integrador al respecto. ${ }^{12}$

El programa del $1^{\circ}$ Encuentro estuvo marcada por una agenda pública política, en la cual se buscó visibilizar problemáticas comunes de las trabajadoras y a su vez cómo conjugar esfuerzos para ocupar lugares de decisión en las mesas políticas de los sindicatos. En este espacio las mujeres pudieron establecer algunas demandas en la agenda pública sindical provincial y nacional: emergencia en violencia de género y femicidios es el reclamo urgente que las Mujeres de ATE Mendoza manifiestan en cada jornada de lucha a gritar ¡Vivas y desendeudadas nos queremos!; el respeto por los derechos humanos de las compañeras y grupos

11 http://www.laizquierdadiario.com/8M-en-Mendoza-Licencia-por-violencia-de-genero-para-lastrabajadoras-de-la-educacion.

12 https://www.atemendoza.org.ar/abrimos-la-invitacion-al-primer-encuentro-nacional-de-mujereslideres-sindicalistas-y-de-las-organizaciones-sociales/. 
trans en todos los ámbitos y sectores; y la importancia de incluir la diversidad en estos aspectos de mujeres.

En 2019, ATE Mendoza incorporó asesoramiento profesional en Violencia de Género y Laboral con personal capacitado con perspectiva de género para el abordaje de las violencias al reconocer que dentro del Estado Provincial se dan dos tipos de violencia: laboral y de género, y que atraviesa a mujeres y varones. En mayo de 2020, buscan visibilizar casos de agresiones y amenazas sistemáticas a mujeres trabajadoras de su sindicato y acompañan la denuncia de mujeres de otros sindicatos.

El 8 de marzo de 2020, culminó una Convivencia en Vendimia de Mujeres de ATE y CTA Autónoma Mendoza que elaboró un Documento con algunas conclusiones que reclaman por el acceso a trabajos dignos bien remunerados y en blanco que le permiten a la mujer empoderarse y lograr la independencia económica que necesita para poder independizarse y no ser sometida ni obligada a permanecer en ámbito de violencia. Se reclama al gobierno por la igualdad y equidad, el cumplimiento de la ley Micaela ${ }^{13}$; refugios para mujeres; permitir la participación con poder de decisión, que todas las mujeres puedan ocupar cargos direccionales y sus ideas y proyectos sean escuchados.

Es oportuno mencionar que ATE es uno de los únicos gremios de los/as trabajadores estatales en Mendoza que incorpora una cláusula de género en la paritaria provincial de este año.

\subsection{LA POLITICIDAD CON ENFOQUE DE GÉNERO}

En relación a otra de las categorías teóricas con las cuales abordamos el trabajo, pensamos que la politicidad con enfoque de género brinda herramientas para indagar en los ámbitos sindicales la significación de lo que es hacer política. El aporte teórico del concepto posibilita reconocer en la práctica de las mujeres sindicalistas un movimiento de visibilización-articulación-consensosapropiación como estrategias de construcción política que interpelan al patriarcado y las jerarquías sindicales.

Hay una politicidad emergente en las prácticas de las mujeres: en la militancia, en la acción colectiva, en la articulación de demandas que excede los canales habituales que conducían al paro o movilización por reclamos salariales, apertura de partidarias, defensa de fuentes de trabajo, etc. Las mujeres han planteado al menos un tema en las agendas de genero de cada uno de los sindicatos - ejemplo la violencia laboral en ATE o la reglamentación de la licencia

13 Ley nacional 27.499/19. Establece la capacitación obligatoria en género y violencia de género para todas las personas que se desempeñan en la función pública, en los poderes Ejecutivo, Legislativo y Judicial de la Nación e invita a adherir a las provincias. Se llama así en conmemoración de Micaela García, una joven entrerriana de 21 años, militante del Movimiento Evita, que fue víctima de femicidio en manos de Sebastián Wagner. Para ampliar ver https://www.argentina.gob.ar/generos/ley-micaela\#. 
por violencia de género en SUTE - y han articulado entre estos dos sindicatos y otros sindicatos provinciales una agenda común en torno a reclamos y denuncias por violencia de género y laboral y condiciones laborales cada 8 de marzo y 3 de junio desde 2018.

En este sentido, las estrategias desarrolladas por las mujeres que han sido referentes en fijar estas agendas de género le van otorgando un significado a su práctica y militancia: la mujer ocupa un lugar central en la reivindicación de derechos laborales asociados en este primer momento a cómo se plantea la violencia contra la mujer. No sólo es un slogan la demanda y rechazo a la violencia de género - que se vivía como algo particular de cada compañera trabajadora sino pensarlo como una problemática laboral, política, social y también hablar y reclamar por la violencia institucional y laboral.

De la misma manera, también resignifican el poder político. Las mujeres trabajadoras reconocen su militancia desde que ingresan al sindicato o acompañando protestas, su parte combativa, el estar en las calles, en las movilizaciones junto a las bases pero no en la toma de decisiones y en los ámbitos de negociación colectiva.

Ahora bien, la politicidad con enfoque de género implica también repensarla en la relación entre trabajo productivo y reproductivo, hoy más que nunca lo personal es político, de lo contrario no hay tiempo concreto-real para la participación política sindical. El ámbito familiar doméstico sigue siendo considerado como propio de mujeres y esto limita la posibilidad de participación, lo cual requiere de políticas estatales y de cambios culturales que permitieran compatibilizar ambos espacios. La desconstrucción de roles y las políticas de compatibilización son imprescindibles para asegurar la participación política de todas, sino la participación queda subsumida a la clase, estado civil, ciclo de vida de las mujeres (ya como madres o a cargo del cuidado de personas).

\section{LEYENDO ALGUNOS DATOS PARA CONTEXTUALIZAR LA RELACIÓN GÉNERO- TRABAJO-SINDICATOS}

\subsection{TRABAJO PRODUCTIVOY REPRODUCTIVO}

La estructura patriarcal de la sociedad ha adjudicado tradicionalmente a las mujeres las mayores responsabilidades en el ámbito doméstico y del cuidado familiar, en tanto que a los hombres se les ha asignado las tareas de protección y provisión de sustento económico del hogar. La construcción de dichos roles ha generado desigualdad entre géneros y en términos económicos invisibilizan y dificulta la medición de las actividades reproductivas que son tan fundamentales como el trabajo para el sostenimiento de una sociedad. Por ejemplo el costo 
invisibilizadas del trabajo no rentado de las tareas domésticas y de cuidados de niños y ancianos al interior del hogar, que recae en las mujeres de las familias ${ }^{14}$. Este costo no se cuantifica dado que es complejo atribuirles un valor monetario a las acciones de cuidados. La vida cotidiana de todos los seres humanos se sustenta en un sin número de acciones que se desarrollan en el ámbito doméstico y que posibilitan desde el alimento hasta el sostén emocional para el mantenimiento de la vida. Gran parte de estas tareas son desempeñadas por las mujeres del hogar y estas acciones de cuidado son conceptualizadas como trabajo reproductivo y que no está remunerado por un salario, es preeminentemente femenino y se presenta solapadamente entre las mujeres.

Nuestro objeto de estudio nos introduce necesariamente en la reflexión respecto a que las mujeres insertas en trabajos productivos se encuentran inmersa en lo que autoras como Balbo (1978) conceptualizan como situaciones de doble presencia, que da cuenta de estrategias cotidianas que las mujeres deben realizar para compatibilizar su actividad laboral con el trabajo doméstico y de cuidados en tiempos que son a su vez sincrónicos. Izquierdo (2003), en este mismo sentido señala que sería tal vez más adecuado hacer referencia a una doble presencia-ausencia por la dificultad de compatibilizar durante mucho tiempo las obligaciones laborales y de cuidados. Esta realidad en el caso que estudiamos se complementa con la participación activa en la vida sindical ligada a su trabajo productivo.

Vamos a comenzar nuestra descripción haciendo referencia a los tiempos que las mujeres dedican al trabajo reproductivo y de cuidados y su falta de cuantificación estadística. El Informe "Las brechas de género en la Argentina Estado de situación y desafíos" (2020) explica claramente cómo la invisibilización de los costos del trabajo reproductivo acentúa la desigualdad de género.

El punto de partida para exponer el lugar de las mujeres en el sistema productivo es un concepto básico y fundamental de la economía: el trabajo. En general, la definición de trabajo que aparece contenida tanto en las estadísticas como en el diseño de las políticas públicas como una actividad mediada por un pago y deja fuera del análisis económico al trabajo doméstico y de cuidados no remunerado que se realiza en los hogares. Esta dimensión que, en general, no se considera a la hora de pensar las condiciones y posibilidades de empleo de las personas, hace que la desigualdad se reproduzca y amplíe. Por ello, incorporar al análisis la distribución social de estos trabajos no remunerados y los roles de género que se reproducen socialmente es clave para entender las desigualdades que luego se captan en los indicadores laborales y

14 Según la Encuesta sobre Trabajo No Remunerado y Uso del Tiempo (EAHU-INDEC, 2013) ellas realizan el $76 \%$ de las tareas domésticas no remuneradas. El 88,9\% de las mujeres las realizan y dedican a este tipo de labores un promedio de 6,4 horas semanales. Mientras tanto, sólo el 57,9\% de los varones participa en estos trabajos, a los que les dedican un promedio de 3,4 horas semanales. 
que sí aparecen de manera visible en gran parte de las estadísticas e información sobre la cuestión (INFORME, 2020).

Este año, se publicó el Informe "Los cuidados, un sector económico estratégico Medición del aporte del Trabajo doméstico y de cuidados no remunerado al Producto Interno Bruto" el cual señala que el Trabajo Doméstico y de Cuidados No Remunerado (TDCNR) sigue quedando al margen del debate económico. El dato contundente es que el TDCNR representa un 15,9\% del PIB argentino y es el sector de mayor aporte en toda la economía, seguido por la industria $(13,2 \%)$ y el comercio (13,0\%). El aporte por género del TDCNR al PIB es desigual: el $75,7 \%$ proviene de tareas realizadas por mujeres. Es decir, las mujeres realizan más de las tres cuartas partes del TDCNR y, de este modo, dedican, el total de ellas en su conjunto, 96 millones de horas diarias de trabajo gratuitas a las tareas del hogar y los cuidados. (INFORME, 2020, p. 11).

El trabajo reproductivo no solamente no es cuantificado en las cuentas públicas, no está remunerado mediante un salario, es un trabajo eminentemente femenino y permanece invisible incluso a los ojos de las personas que lo llevan a cabo. Esta dedicación no es idéntica para todas las mujeres, porque varía según los distintos momentos de su ciclo de vida y según su clase social. Aumenta si se vive en régimen de dedicación exclusiva o de doble presencia. En nuestro caso de estudio la situación de doble presencia y activa participación sindical nos lleva nuevamente a repensar la necesidad de una politicidad con enfoque de género que dé cuenta de la situación en las cuales las mujeres deben luchar por obtener visibilización y poder al interior de sus sindicatos.

En cuanto al trabajo productivo, nos parece oportuno realizar una breve descripción y sistematización sobre algunos datos del mercado laboral y la participación de las mujeres en el mismo tanto en el país como en Mendoza. Si bien los trabajos a los que recurrimos tienen diferentes formas de medición, todos ellos muestran tendencias de largo plazo.

En Argentina, desde los años 90 se evidencia un crecimiento en la tasa actividad de las mujeres, en relación a los 70 . Eliana Aspiazu (2015) sostiene que a pesar de esta mayor inclusión en el mercado de trabajo, las desigualdades en materia de inserción laboral de las mujeres persisten y el mercado de trabajo sigue presentando profundos desafíos: las tasas de actividad y de empleo continúan siendo todavía hoy muy inferiores en las mujeres; en tanto que las tasas de empleo no registrado femeninas se mantienen en niveles superiores a las masculinas. A estas desigualdades hay que sumar que aún persisten importantes brechas salariales y mecanismos de segregación y discriminación que producen una mayor concentración de las mujeres en ciertas ramas de actividad vinculadas a los servicios, es también reconocida la dificultan de acceso a los puestos de mayor jerarquía tanta en el ámbito del empleo estatal como privado.

La aceleración del aumento de la tasa de actividad femenina en los $90^{\circ}$ se explica fundamentalmente por la activación de las mujeres de hogares de 
ingresos bajos y medios bajos y se enmarca, por tanto, en una estrategia orientada a sortear los efectos del desempleo y la precarización de los jefes de hogar.

El dato respecto a la tasa de participación de las mujeres en el mercado laboral o tasa de actividad es un indicador que da cuenta no solo de la menor participación laboral en comparación a los varones, sino también de las desiguales y en muchos casos precarias condiciones en las cuales se produce la inserción laboral femenina.

\begin{abstract}
Al tener una cantidad de tiempo disponible menor, las mujeres participan menos en el mercado de trabajo. Además, se insertan laboralmente con peores condiciones: salarios más bajos, doble jornada (paga y no paga), mayor precarización, altas tasas de desempleo, pobreza de tiempo, entre otras. La tasa promedio de la participación de las mujeres en el mercado laboral es de 49,2\%, 21 puntos porcentuales más baja que la de los varones $(71,2 \%)$. Hay diferencias entre las mujeres también. Según datos del INDEC, las que cuentan con estudios universitarios completos o incompletos tienen una tasa de actividad de $74,3 \%$ mientras que las que poseen secundario incompleto muestran una participación del 35,9\% y las que poseen estudios secundarios completos 55,1\% (INFORME BRECHA DE GÉNERO, 2020).
\end{abstract}

A partir del 2003, la incorporación de las mujeres al mercado de trabajo evidencia una leve disminución, pero los niveles de participación siguen en aumento y muestran brechas en la tasa de actividad, respecto del hombre que se mantienen estables en el tiempo ${ }^{15}$. La tasa de ocupación, por su parte, tiene una evolución positiva - tanto para hombres como para mujeres - y la brecha de género, si bien muestra una persistente desigualdad, mejoró significativamente, dado que en 2012 la brecha fue de $-35,6 \%$, mucho más baja que la que existía en los primeros años de la década del 90 'en la cual era del orden del -50\%. La brecha existente en niveles de desempleo era para el 2003 del 25,8\% hasta llegar al $41,7 \%$ en 2012 .

\title{
3.2. APROXIMACIÓN A ALGUNOS DATOS SOBRE TRABAJOY GÉNERO EN MENDOZA
}

Si bien podemos establecer generalidades y tendencias a partir de los datos nacionales, nos parece oportuno presentar y sistematizar algunos datos

15 Cabe destacar que no es posible la comparación de los valores de las tasas de actividad de períodos anteriores y posteriores a mayo de 2003 debido a cambios metodológicos introducidos en la EPH (que involucran una mejor captación tanto de la población ocupada como de la desocupada y, por lo tanto, de la tasa de actividad). Por ese motivo, se exponen los resultados en cuadros separados. 
sobre mercado laboral y género, sobre la inserción laboral de las mujeres y su especificidad en nuestra provincia.

La producción estadística con perspectiva de género es escasa y si bien reconocemos algunos trabajos (ALONSO, 2012, 2017, 2019; MARTIN, 2013, 2019; BURGARDT, 1999, 2004, 2007; BURGARDT, IZURA, NERI y SANCHO 2004; DALLA TORRE, DI COSTA y NERI, 2006; DALLA TORRE, 2008) estos se concentran en análisis por rama de actividad, temáticas específicas y aquellos que abordan en general la temática de trabajo y mujer han sido discontinuados.

Rescatamos tres fuentes estadísticas que contextualizan nuestro periodo bajo estudio y nos brindan un acercamiento al mercado laboral y mujer: a) el Informe de Diagnostico Laboral 2012-2016 ${ }^{16}$; b) Informe sobre la desigualdad de género en el mercado laboral 2003-2014 en base a datos de la Encuesta Permanente de Hogares (INDEC); c) análisis de las Encuestas de condiciones de Vida 2016-2019 realizada por la Dirección de Estadísticas y Censo de Mendoza.

a) Según datos de la Encuesta Permanente en la provincia de Mendoza se encontraban activas, al 4to trimestre de 2016, 25.905 empresas en todos los sectores de la economía. Se destacan, por su peso relativo, las empresas del sector agropecuario, cuya presencia en el tejido productivo de esta región casi duplica a la que se observa a nivel nacional. Algo similar sucede con las firmas dedicadas a la minería y petróleo. La composición de las empresas por tamaño muestra algunas diferencias en relación a la estructura nacional. En primer lugar, se destaca la existencia de una mayor proporción de firmas grandes y pymes. Como contrapartida se observa una menor presencia de microempresas:

En este contexto ¿qué sucede con las mujeres y varones en el sector privado?

- Desde una perspectiva de mediano plazo, entre 2012 y 2017 el empleo registrado presentó un aumento del $0,3 \%$, lo que representa la creación neta de 700 puestos de trabajo registrados. A nivel nacional se registró un aumento superior (3,5\%).

- A nivel sectorial, este aumento del empleo se debió principalmente al comercio $(4,7 \%)$ y a los servicios $(5,5 \%)$. En conjunto estas actividades determinaron la creación de más de 7,8 mil puestos de trabajo en el período.

- Las mujeres, al igual que los jóvenes, suelen tener una inserción laboral más precaria que sus pares varones, mostrando mayores tasas de desempleo, subempleo y empleo no registrado.

- Adicionalmente, suelen presentar menores tasas de actividad, dando cuenta del rol social que asume este colectivo, con una amplia dedicación al cuidado del hogar y las personas dependientes.

- En relación con las mujeres de entre 15 y 65 años del total relevado, aquellas que residen en el aglomerado provincial, presentaron durante el primer trimestre de 2018 una tasa de empleo similar al total de los aglomerados y una tasa de actividad más elevada.

16 Informe sobre Mendoza, Ministerio Trabajo, Empleo y Seguridad Social Subsecretaría de Planificación, Estudios y Estadísticas. 2019. 
- Respecto a los varones de su jurisdicción, las mujeres estuvieron más afectadas por los bajos niveles de actividad y empleo, mientras que tuvieron tasas más elevadas de desocupación, subocupación y empleo no registrado.

- En el año 2017 el empleo femenino en Mendoza representa el 29,2\% del total del empleo privado registrado en la provincia. Esa relación está tres puntos por debajo de la participación de las mujeres en el total del empleo registrado del país $(32,6 \%)$.

- La estructura del empleo femenino de Mendoza refleja su perfil de especialización. Se registra el elevado peso que tiene el empleo del sector primario $(6,2 \%$ de las trabajadoras) en la provincia respecto al total nacional $(2,2 \%)$.

- La brecha salarial entre hombres y mujeres en Mendoza es del 25,6\%. A su vez, dicha brecha cayó desde el 2012. En el 2012 las mujeres ganaban el 29,5\% menos que los hombres, y en el 2017 perciben remuneraciones en promedio $25,6 \%$ menores que los hombres.

b) en cuanto a la inserción laboral por sexo y la desigualdad de género en el mercado de laboral:

- La tasa de actividad femenina, si bien es más baja que la nacional, aumenta levemente durante $2003-2014$ del $43,6 \%$ al $45,9 \%$ y la brecha de genero se reduce alcanzando el $34 \%$.

- También crece la tasa de empleo femenino que pasa de 36,6\% en 2003 a $43,2 \%$ en 2014. Pero, la tasa de desempleo de las mujeres continúa por encima de la masculina en todo el periodo.

- En términos de subocupación, entre 2004 y 2013, la participación de las mujeres en la subocupación crece en mayor medida que en el total de los aglomerados urbanos, al tiempo que aumenta la proporción de mujeres como subocupadas demandantes. Si bien el lapso cierra con mejoras en términos de aumento del empleo y reducción de la desocupación, se acentúa la subocupación femenina, especialmente la demandante:

c) La Encuestas de Condiciones de Vida, nos posibilita acceder a datos sobre los conglomerados urbanos y rurales en los últimos cuatro años (2016-2019) y nos brinda información del mercado laboral que es significativa.

- Crece la ocupación de las mujeres entre 2016 y 2019 por lo que la brecha de género se sostiene en un $0,6 \%$ promedio en este periodo. Si bien en 2016 se da el menor porcentaje de inactividad ${ }^{17}$ de las mujeres, la tendencia se sostiene. Es cuando observamos los datos relativos a la desocupación donde está se acentúa.

- En cuanto al análisis de la categoría ocupacional, la brecha de género es menor en la categoría cuentapropista y obrero/empleado. En cambio, se profundiza cuando se analiza "patrón" y "trabajo familiar". El porcentaje de patrones varones duplica a las mujeres; sin embargo cuando miramos la categoría trabajador familiar, son las mujeres sobre las que recaen las tareas regulares de ayuda en la actividad laboral de un familiar.

17 Inactivo: Persona que no participa en la actividad económica es decir, la que no tiene trabajo ni lo busca activamente. 
Si bien la brecha de género se mantiene dentro de los mismos parámetros en las actividades primarias y secundarias, la diferencia más significativa se da en las actividades terciarias. Sin embargo, una observación por cada una de las ramas de actividad nos lleva a poner el foco en la dimensión "zona urbana o rural” y la brecha de género se profundiza más en la zona urbana que rural.

\subsection{MAPEANDO LOS SINDICATOS EN MENDOZA CON PERSPECTIVA DE GÉNERO}

En Mendoza hay setenta y nueve sindicatos registrados y cuarenta y nueve con inscripción gremial según datos del Registro Ministerio de Trabajo, Empleo y Seguridad Social de la Nación. La dispersión geográfica sindical coincide con que la mayor cantidad de población se encuentra en el Gran Mendoza (Capital; Lujan; Godoy Cruz; Guaymallén; Maipú y Lavalle).

La distribución espacial es la siguiente ${ }^{18}$ :

- Gran Mendoza: 96 (83 en Capital; 2 Lujan; 2 Godoy Cruz; 3 Guaymallen; 3 Maipu; 1 Lavalle).

- Este: 14 (3 Rivadavia-Junín-Santa Rosa; La Paz; 2 en Palmira; 6 en San Martin).

- Sur: 15 (5 en Gral. Alvear; 6 en San Rafael; Malargüe, Villa Atuel, $4^{\circ}$ distrito).

- Valle de Uco: 6 (2 en San Carlos; 2 en Tunuyán; 1 Valle Uco; 1 Tupungato).

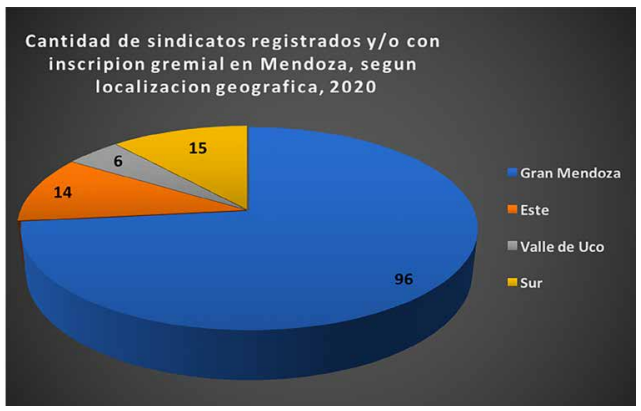

Fuente: Elaboración propia en base a datos del Ministerio de Trabajo, Empleo y Seguridad Social de la Nación.

18 La distribución espacial se toma según el domicilio de la sede sindical declarado en el Registro Ministerio de Trabajo, Empleo y Seguridad Social. 
En cuanto a la rama de actividad a la que pertenecen los sindicatos, catorce son sindicatos pertenecientes a actividades primarias; catorce son parte de la actividad secundaria y cien sindicatos de actividades terciarias.

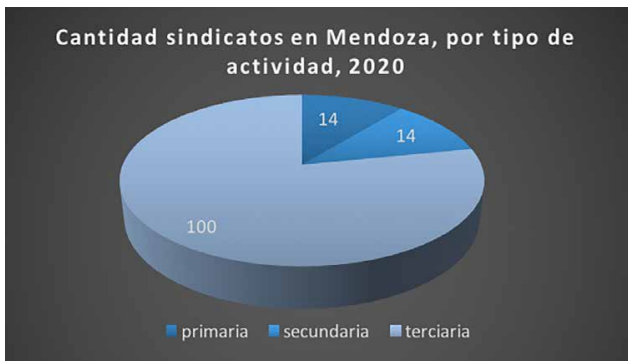

Fuente: Elaboración propia en base a datos del Ministerio de Trabajo, Empleo y Seguridad Social de la Nación.

Del relevamiento realizado nos preguntamos cuantos sindicatos tiene en su ámbito de toma de decisiones - secretaria general o secretaria adjunta - una mujer; cuantos sindicatos tienen un área institucional de la mujer, de género, de igualdad de género, y/o diversidad según el nombre que adquiera en cada sindicato pero que sea específicamente de género y no se funda con áreas de acción social, turismo y/o familia como habitualmente sucede; y por ultimo nos preguntamos cuantos sindicatos en donde hay una mujer en el área de toma de decisiones tiene secretaria de la mujer. Los resultados que obtuvimos son los siguientes:

- En sólo el 14,4\% de los sindicatos de Mendoza, una mujer es secretaria general o secretaria adjunta.

- En el 12,2\% de los sindicatos estatales de Mendoza, una mujer es secretaria general $o$ adjunta.

- Del total de los sindicatos en Mendoza, solo el 11\% tiene una secretaría de la mujer, de género y/o diversidad.

- En los sindicatos donde una mujer ocupa el área de toma de decisiones, solo el 23\% tiene área de la mujer, de género y/o diversidad.

\section{ALGUNAS REFLEXIONES SOBRE EL PROCESO DE INVESTIGACION}

Desde la perspectiva interpretativa, crítica y del punto de vista feminista, resulta muy pertinente trabajar desde la metodología cualitativa, ya que son 
compatibles los diferentes métodos para trabajar en diversas disciplinas, y permite una mayor profundidad con un menor número de casos, se logra un mayor acercamiento a cada uno/a de los/as participantes de la investigación y se mueven emociones y afectos entre ellos (RÍOS, 2012, p. 189).

Nuestra investigación es de corte cualitativo recurriendo a técnicas como entrevista en profundidad, semiestructuradas, análisis documental y bibliográfico y observación-participación en diferentes instancias de los espacios sindicales y momentos de movilización en las calles. El mismo proceso de investigación nos ha generado algunos retos: por la necesidad de ir construyéndolo al tomar un enfoque de género y por la pandemia; por algunas contradicciones teóricometodológicas; por la interrelación que se da en cada una de nosotras como investigadoras, militantes, mujer; por el diálogo entre teoría y prácticas y los condicionantes propios de los espacios sindicales que forman parte de nuestra unidad de análisis.

En toda investigación feminista se considera siempre, de forma explícita o no, la división social por géneros y se contempla su jerarquía. De acuerdo con la forma en que se explique la división genérica de la sociedad y las posibles soluciones ante la supremacía del género masculino, se darán las variantes dentro del feminismo o lo que se ha dado en llamar distintos feminismos.

Sin embargo, a pesar de las diferencias, existen ciertos elementos en común, a los que se puede denominar "punto de vista feminista" aun cuando se trate de distintos feminismos y, por lo tanto, de "distintos puntos de vista feministas" (BARTRA, 2012, p. 70).

Se puede hacer investigación sobre mujeres e investigación feminista diferentes entre sí y hay múltiples maneras de hacer una y la otra. En la investigación feminista se ha utilizado una multiplicidad de ellas pero, por ejemplo, en algunas ciencias sociales se ha privilegiado la llamada entrevista semiestructurada, y frecuentemente se recurre a la historia oral. En las ciencias sociales y en las humanidades al tomar en cuenta el género, se ha manifestado gran interés por estudiar específicamente a las mujeres, que hasta hace poco eran básicamente invisibles, como actoras sociales (BARTRA, 2012, p. 71).

Es preciso señalar que no se trata de que las mujeres, por el hecho de serlo, realicen necesariamente un tipo de investigación distinta con una metodología diferente. Un punto de vista feminista puede dar lugar a una metodología de investigación distinta y resulta pertinente denominarla feminista.

En este sentido, pensando el punto de vista feminista durante el trabajo de campo en cuanto a la observación-participación y el momento de entrevista sostenemos que durante la observación - participación en las movilizaciones (antes de la pandemia) no es neutra, en ellas han influido la sensibilidad ante el reclamo y la necesidad de establecer una vigilancia metodológica al retratar el momento - por ej. en marchas de \#8M o \#NiUnaMenos-, realizar entrevistas, escuchar discursos o lectura de documentos. 
En relación al momento de las entrevistas, el hecho de ser mujer también condiciona el proceso de investigación, no es lo mismo que acuda un investigador a realizar entrevistas a varones que sea una mujer quien va a entrevistar varones. En nuestro caso, por ejemplo, hay sindicatos a los que se han acercado varones a hacer entrevistas y en aquellos en los que hemos ido mujeres nos han dicho cosas diplomáticamente correctas y algunos entrevistados reconocen - en off - "les vamos a decir lo que quieren escuchar". De la misma forma, una mujer entrevistando (aún no siendo feminista) o acompañando en algunas presentaciones a otras mujeres establece otro tipo de relación. Y en este sentido, pensamos que es la variable "género" más que la clase social, la posición política o académica lo que más ha influido en esta instancia de la investigación.

La visión de género implica reconocer que socialmente existe un conjunto de ideas, representaciones y creencias basadas en que hay cosas propias de hombres y de mujeres. Pero esta separación y distinción de papeles masculinos y femeninos provoca la participación diferenciada, jerárquica y desigual dentro de las instituciones sociales, políticas y económicas. Por ello, los estudios de género se centran en los/as sujetos/as, y en la manera en que la cultura capitalista patriarcal expresa las diferencias entre ellos; es decir, en la construcción de condiciones culturales simbólicas y subjetivas responsables de la reproducción de ciertas ideologías de poder y opresión, generalmente de los hombres hacia las mujeres.

Las teorías crítica feminista y del punto de vista implican posturas políticas que ubican la relación teoría-práctica como base fundamental para la transformación. Se proponen descubrir los dispositivos y mecanismos de orden social y patriarcal que posibilitan desigualdades de género entre hombres y mujeres, con el propósito de gestar nuevas relaciones, toma de conciencia y formas de emancipación (RÍOS, 2012, p. 191).

En nuestro proceso investigativo seguimos algunas pautas dadas por Ríos (2012, p. 192), comoflexibilidad para volver al trabajo de campo para la recolección de datos, ajustes de la información en las entrevistas o recopilar nuevos datos que complementen la investigación. Informar el propósito, objetivos y preguntas de investigación con la mayor apertura y responsabilidad ética, respecto de las consecuencias que la actividad de observar, indagar, entrevistar e interpretar puede provocar sobre quienes van a participar. Cuidar la confidencialidad y fundamentalmente dejar de lado la relación vertical entre el/la investigador/ra $y$ los objetos de investigación, la visión desde arriba para remplazarla por una conveniente visión desde abajo.

La decisión de participar activamente en las acciones, movilizaciones de las mujeres está presente en nuestra investigación, y si bien se han visto afectadas por la situación de pandemia, esta acción la seguimos en las redes a través de las cuales se ha generado una movilización colectiva en los sindicatos bajo estudio y en la Mesa Intersindical de Mujeres - Mendoza. En este sentido, se hace necesario colectivizar las experiencias propias de investigación, socializar con mujeres, 
varones, colectivos disidentes los pareceres que vertimos en la investigación para ser comprendidos y que nos permitan reconocer y (re)significar - en la reflexión teórica y metodológica - las desigualdades de género en la práctica política sindical como aquellas situaciones naturalizadas en las relaciones jerárquicas patriarcales en los ámbitos sindicales y laborales.

\section{A MODO DE CIERRE}

Las siguientes consideraciones sobre el objeto de estudio abordado en este artículo de ninguna manera cierran el tema, nos parece un proceso abierto de investigación-observación-práctica y reflexión. Así, en cuanto a lo conceptual, nuestra mirada desde la categoría género nos ha permitido comprender las demandas y disputas en la conformación de la/s agenda/s de género sindicales como también indagar en las identidades de las trabajadoras que se van conformando en las relaciones de poder dentro de los sindicatos y en la interrelación sindicatos-gobierno. Las trabajadoras en lo sindicatos están construyendo un sentido de politicidad sobre cómo hacer política en la confluencia de diferentes espacios como el laboral, el de militancia sindical o intersindical, o los ámbitos de conducción y/o decisión sindical que recae en cada una de ellas.

En cuanto a la producción de datos con perspectiva de género es incipiente en nuestro país, son escasos los estudios con alcance nacional que relevan datos económicos, sociales, laborales con esta perspectiva. La pandemia de 2020 ha puesto de manifiesto la necesidad de medir y conocer las tareas de cuidado, de sacar a la luz el trabajo productivo y reproductivo de mujeres y colectivos disidentes.

Mendoza no escapa a esta situación; sin embargo, desde 2015 se releva la Encuesta de Condiciones de Vida la cual pone la mirada en educación, ocupación, actividad económica, salud, distribución de la población con perspectiva de género y también con la dimensión de "zona urbana o rural".

En cuanto a los sindicatos, carecen de relevamientos estadísticos, es difícil en ocasiones conocer algún dato básico como cuántos afiliados/as tiene cada sindicato. Por ello, nos parece sumamente importante la producción de datos que realizamos con perspectiva de género la cual nos permitió conocer que en sólo el 14,4\% de los sindicatos de Mendoza, una mujer es secretaria general o secretaria adjunta; y cuando nos referimos a sindicatos estatales, solo en el 12,2\% de ellos, una mujer es secretaria general o adjunta. Del total de los sindicatos en Mendoza, solo el $11 \%$ tiene una secretaría de la mujer, de género y/o diversidad y en aquellos sindicatos donde una mujer ocupa el área de toma de decisiones, solo el 23\% tiene área de la mujer, de género y/o diversidad. Los datos hablan y son una fotografía de una situación estructural que llevara tiempo revertir e igualar.

Por ello, los rasgos del nuevo tiempo y la/s agenda/s de género visibilizan desigualdades históricas en las tareas de cuidado, en los déficits de condiciones 
laborales con perspectiva de género, en las brechas salariales en el trabajo productivo y reproductivo, en las brechas de representación en la estructura organizacional que va desde la existencia de áreas de género hasta la igualdad en la distribución de cargos de poder y decisión; en las violencias hacia las mujeres; en los mecanismos de promoción de la igualdad en los espacios sindicales; y en la construcción y temas de agenda (las agendas).

En este trabajo nos acercamos a la/s agenda/s de género desde las miradas de los géneros y sus relaciones de poder. Pensamos que la revitalización sindical ha tenido una cara de género también, lo cual conduce a pensar una politicidad femenina con especificidades de construcción y acción política que implica una reflexión teórica y práctica. El mundo sindical está siendo modificado y revolucionado desde sus bases por una marea feminista que ponen en cuestión un orden patriarcal/capitalista/ desigual dominante y también jaquea y cuestiona prácticas sindicales reificada, batallando por espacios concretos de poder, reconocimiento y deconstrucción de una institución jerárquica y tradicionalmente patriarcal como el sindicalismo en Argentina y Mendoza.

Pensar la politicidad con enfoque de género y vivir sus prácticas sindicales implica observar dialécticamente la dimensión productiva y reproductiva del mundo del trabajo. Implica cuestionar las tareas reproductivas de las mujeres y los tiempos que ello implica de cuidado que debilitan la participación social y política de las mujeres en espacios productivos. En los espacios productivos sindicales la politicidad reconoce un movimiento de visibilización-articulaciónconsensos-apropiación como estrategias de acumulación de fuerza política para la acción colectiva y para el acceso a espacios de poder en la organización sindical.

El ámbito familiar doméstico sigue siendo considerado como propio de mujeres, este se vuelve un limitante un mecanismo que limita la posibilidad de participar; lo que requiere de políticas estatales que permitan compatibilizar los espacios y de cambios culturales al interior de la familias. La desconstrucción de roles y las políticas de compatibilización son imprescindibles para asegurar la participación políticas de todas, sino la participación es según clase, estado civil, ciclo de vida, madre o a cargo de cuidado de personas.

En cuanto al análisis de la/s agenda/s consideramos que hay una agenda en común que han podido ir construyéndose como la de Mujeres Sindicalistas de la Corriente Federal de los Trabajadores de la Central General de Trabajadores (CGT), la Mesa Intersindical de Mujeres en Mendoza, y agendas de género de cada sindicato.

Nuestro programa de investigación teórico práctico estaba planteado a partir de una metodología cualitativa a través de búsqueda de información, lectura teórica, observación y participación en diferentes instancias de acción política y movilización de los sindicatos bajo estudio, que se han visto alterada por la situación de pandemia, lo cual nos ha hecho volver sobre las acciones a través de las redes sociales y que nos abre nuevos interrogantes acerca de la oportunidades y debilidades de llevar a cabo una investigación a través de estos medios. Por lo 
cual coincidimos con Eli Bartra (2012, p. 75) que como cualquier otro método, el feminista no está creado de antemano, se va creando en la medida que se desarrolla el trabajo investigativo.

\section{REFERÊNCIAS}

ALBERDI, Inés. El significado conexión entre el ser y el pensar del género en las ciencias sociales. Revista Política y Sociedad. Universidad Complutense de Madrid, 32, 1999, pp. 9-21.

ALONSO, Virginia. Una aproximación al análisis de la desigualdad de género en el mercado de trabajo mendocino durante la posconvertibilidad. En GRUPO A: DINÁMICA DEL MERCADO DE TRABAJO. DISTRIBUCIÓN DEL INGRESO Y EVOLUCIÓN SALARIAL. Mendoza: ITP-UNCUYO / CONICET, 2014.

AQUIN, Nora. La politicidad de sectores populares: politicidad popular: estrategias de vinculación social y política de sectores de pobreza urbana. Córdoba: Universidad Nacional de Córdoba, 2018.

ASPIAZU, Laura. Participación de las mujeres e institucionalidad de la problemática de género en el sindicalismo argentino. En 12 CONGRESO NACIONAL DE ESTUDIOS DEL TRABAJO, Buenos Aires, 2015.

BARRIOS, María José. El espacio de la mujer en los sindicatos municipales. Periodicas.com.ar, 2020. Disponible en https://periodicas.com.ar/2020/01/23/ el-espacio-de-la-mujer-en-los-sindicatos-municipales/.

BARTRA, Eli. Acerca de la investigación y la metodología feminista. En BLAZQUEZ GRAF, Norma; FLORES Fátima; PALACIOS, Maribel ( coord.). Investigación feminista : epistemología, metodología y representaciones sociales. México : UNAM, Centro de Investigaciones Interdisciplinarias en Ciencias y Humanidades : Centro Regional de Investigaciones Multidisciplinarias : Facultad de Psicología, 2012, pp. 67-77.

BLAZQUEZ GRAF, Norma; FLORES Fátima; PALACIOS, Maribel ( coord.). Investigación feminista : epistemología, metodología y representaciones sociales. México : UNAM, Centro de Investigaciones Interdisciplinarias en Ciencias y Humanidades: Centro Regional de Investigaciones Multidisciplinarias : Facultad de Psicología, 2012. 
BONACCORSI, Nélida y CARRARIO, Marta. Participación de las mujeres en el mundo sindical. Un cambio cultural en el nuevo siglo. En Revista La Aljaba, Universidad Nacional del Comahue segunda época, Volumen XVI, 2012.

CONTARTESE, Daniel y MACEIRA, Verónica. Informe Trabajo, ocupación y empleo. Relaciones laborales, territorios y grupos particulares de actividad Diagnóstico sobre la situación laboral de las mujeres. En Documento 136 Trabajo, ocupación y empleo. Relaciones laborales, territorios y grupo particulares de actividad, Buenos Aires, Ministerio de Trabajo, 2005.

DEIE. Informe Encuestas Condiciones de Vida 2016, 2017, 2018 y 2019. Mendoza.

DÍAZ, Estela y SOCOLOVSKY, Yamile. Feminismo y sindicalismo, la potencia de un encuentro Algunas reflexiones en torno al caso argentino. En GOREN, Nora y PRIETO, Vanesa Lorena (eds) Feminismos y sindicatos en Iberoamérica. Ciudad Autónoma de Buenos Aires: CLACSO, 2020.

GIL, Silvia. Nuevos Feminismos. Madrid: Traficantes de sueños, 2011. Disponible en: http://www.vitoriagasteiz.org/wb021/http/contenidosEstaticos/adjuntos/ es/67/88/46788.pdf.

GOREN, Nora y PRIETO, Vanesa Lorena. Feminismos y sindicatos en Iberoamérica. Ciudad Autónoma de Buenos Aires: CLACSO, 2020.

GORRI, Patricia, LECARO, Patricia y JAUREGUIBERRY, Karina. El sindicalismo estatal en la Provincia de Mendoza en un contexto de ajuste y disciplinamiento. En $8^{\circ}$ CONFERENCIA LATINOAMERICANA Y CARIBEÑA DE CIENCIAS SOCIALES Y PRIMER FORO MUNDIAL DEL PENSAMIENTO CRÍTICO, CLACSO, Buenos Aires, 2018. Disponible en http://www.clacso.org.ar/ conferencia2018/presentacion_ponencia.php?ponencia=2018812174519-6756pg.

GORRI, Patricia, LECARO, Patricia y otros. Informe Final Proyectos de Investigación 2016-2018 sobre Sindicatos y Gobierno: relaciones, dilemas, tensiones con el Nuevo Gobierno. La Intersindical En Mendoza. 2015-2018. Mendoza: Universidad Nacional de Cuyo, 2019.

Informe Encuesta sobre Trabajo No Remunerado y Uso del Tiempo. Argentina: EAHU-INDEC, 2013.

Informe Encuesta de Indicadores Laborales 2019 de Mendoza. Buenos Aires: Ministerio Trabajo, Empleo y Seguridad Social Subsecretaría de Planificación, Estudios y Estadísticas, 2019. 
Informe Las brechas de género en la Argentina Estado de situación y desafíos. Buenos Aires: Ministerio de Economía, 2020. Disponible en: https://www. argentina.gob.ar/sites/default/files/las_brechas_de_genero_en_la_argentina_0. pdf.

Informe TDCNR como parte del PIB 2020. Buenos Aires: Dirección Nacional de Economía, Igualdad y Genero de la Nación, 2020.

KANDEL, Ester. Las mueres dentro de las organizaciones sindicales. Buenos Aires: ARGENPRESS, 2013.

KANTOLA, Johanna. Feminist Theorize the State. Houndmills: Palgrave (cap 1), 2006.

LENGUITA, Paula. Apuntes actuales del sindicalismo argentino. Revista de la Facultad de Ciencias Sociales/UBA, 2017. Disponible en: http://www.sociales. uba.ar/wp-content/blogs.dir/219/files/2017/05/REVISTA93-028-LENGUITA. pdf.

LENGUITA, Paula. La trama feminista en los movimientos sindicales. Revista Estudos do Trabalho Ano IX - Número 23, 2019. Disponible en: www. estudosdotrabalho.org.

LENGUITA, Paula. Género y teletrabajo en pandemia. El País Digital, 2 de agosto de 2020 .

LOMBARDO, Emanuela. Desigualdad de género en la política: un análisis de los marcos interpretativos en España y en la Unión Europea. Revista Española de Ciencia Política. Madrid, 18, 2008, pp. 95-120. Disponible en http://www.ucm. es/info/target/Art\%20Chs\%20ES/ArtPolESUE_RECP_08ES.pdf

NATALUCCI, Ana y REY Julieta., ¿Una nueva oleada feminista? Agendas de género, repertorios de acción y colectivos de mujeres (argentina, 2015-2018). Revista de Estudios Políticos y Estrategicos, Chile, Vol. 6, No2, 2018. Disponible en https://revistaepe.utem.cl/articulos/una-nueva-oleada-feminista-agendas-degenero-repertorios-de-accion-y-colectivos-de-mujeres-argentina-2015-2018/.

PATEMAN, Carole. Críticas feministas a la dicotomía público/privado. En CASTELLS, Carmen comp. Perspectivas Feministas en Teoría Política. Barcelona: Paidós, 1996, pp. 31-52. Disponible en: http://es.scribd.com/doc/120277264/ Carole-Pateman. 
RÍOS EVERARDO, Maribel. Metodología de las ciencias sociales y perspectiva de género. En BLAZQUEZ GRAF, Norma; FLORES Fátima; PALACIOS, Maribel (coord.). Investigación feminista : epistemología, metodología y representaciones sociales. México : UNAM, Centro de Investigaciones Interdisciplinarias en Ciencias y Humanidades: Centro Regional de Investigaciones Multidisciplinarias: Facultad de Psicología, 2012, pp. 179-197.

SILVA PELOSSI, María. Trabajo final de grado: Trabajo femenino y relaciones de género [en línea]. Universidad Nacional de La Plata. Facultad de Humanidades y Ciencias de la Educación, 2006. Disponible en: http://www.memoria.fahce.unlp. edu.ar/tesis/te.660/te.660.pdf.

VILAS, Carlos, El proyecto de Macri es el del capital especulativo. Diario Página 12, 1 de marzo de 2017. Disponible en: https://www.pagina12.com.ar/22974-elproyecto-de-macri-es-el-del-capital-especulativo.

WAINERMAN, Catalina y NAVARRO, Marysa. El trabajo de la mujer en la Argentina: un análisis preliminar delas ideas dominantes en las primeras décadas del siglo XX. Cuadernos del CENEP, No 7, Uruguay, 1979. 
ABSTRACT: The trade union movement is once again the object of study for the Social Sciences, accentuating different dimensions of study with respect to the 90 s, emerging in the last five years the issue of the feminist tide in the trade union sphere. We present this work on the gender agenda / $s$ in the state unions of Mendoza Argentina - which make visible the political and economic reconfiguration that during the 2016-2020 period in which the Cambiemos alliance governs, clearly conservative in its public policies which led to an adjustment in neoliberal terms that affected mostly workers, influencing their organization, representation, mobilization and agendas. This work is a first approach in our research process to the union gender agenda and at the same time allows us to reflect on the dilemmas faced by academic commitment and critical research when approaching our object of study from the conceptual point of view and in its union political practice. The work is structured as follows: first, we make a conceptual journey that accounts for those categories that we consider most fruitful for the analysis and interpretation of our object of study. In this sense, we return to the theoretical path traveled by referents on the subject of feminism and unionism (Gamba, 2008; Aspiazu, 2015; Goldman, 2018; Lenguita, 2017, 2019, 2020; Natalucci y Rey, 2018; Díaz y Socolovsky, 2020; Goren y Prieto, 2020). With these theoretical tools we visit concepts and describe feminist activism in state unions in Mendoza, trying to account for their practices, discourses and agendas. In a second moment, we make a methodological approach by approaching the secondary data on work and gender in Mendoza that allow us to quantitatively size the phenomenon under study. In the third instance, we carried out the production of a mapping of the provincial unions regarding the institutional structures to observe if the gender perspective is visible in their internal organization. Finally, we reflect on the limits and challenges of the research process in times of pandemic.

KEYWORDS: State unions. Gender. Union Gender agenda. Mendoza.

\section{A AGENDA SINDICAL DE GÊNERO EM MENDOZA, ARGENTINA. 2016- 2020}

RESUMEN: O movimento sindical é mais uma vez objeto de estudo para as Ciências Sociais, acentuando diferentes dimensões de estudo no que diz respeito aos anos 90, emergindo nos últimos cinco anos a questão da maré feminista no âmbito sindical. Apresentamos este trabalho sobre a / s agenda / s de gênero nos sindicatos estaduais de Mendoza - Argentina - que tornam visível a reconfiguração política e econômica que durante o período 2016-2020 em que governa a aliança Cambiemos, claramente conservadora em seu políticas públicas, que levaram a um ajuste em termos neoliberais que afetou principalmente os trabalhadores, influenciando sua organização, representação, mobilização e agendas. Este trabalho é uma primeira aproximação em nosso processo de investigação da agenda sindical de gênero e ao mesmo tempo nos permite refletir sobre os dilemas enfrentados pelo compromisso acadêmico e pela pesquisa crítica ao abordar nosso objeto de estudo desde o ponto de vista conceitual e em sua prática política sindical. O trabalho está estruturado da seguinte forma: primeiro, fazemos um percurso 
conceitual que dá conta das categorias que consideramos mais fecundas para a análise e interpretação do nosso objeto de estudo. Nesse sentido, retomamos o percurso teórico percorrido por referentes sobre o tema feminismo e sindicalismo (Gamba, 2008; Aspiazu, 2015; Goldman, 2018; Lenguita, 2017, 2019, 2020; Natalucci y Rey, 2018; Díaz y Socolovsky, 2020; Goren y Prieto, 2020). Com essas ferramentas teóricas, visitamos conceitos e descrevemos a militância feminista nos sindicatos estaduais de Mendoza, tentando dar conta de suas práticas, discursos e agendas. Num segundo momento, fazemos uma abordagem metodológica abordando os dados secundários sobre trabalho e gênero em Mendoza que nos permitem dimensionar quantitativamente o fenômeno em estudo. Na terceira instância, realizamos a produção de um mapeamento dos sindicatos provinciais quanto às estruturas institucionais para observar se a perspectiva de gênero é visível em sua organização interna. Por fim, refletimos sobre os limites e desafios do processo de pesquisa em tempos de pandemia.

PALABRAS CLAVE: Sindicatos estaduais. Gênero. Agenda sindical de gênero. Mendoza. 